\title{
The Effects of Viscosity on the Structure of Shock Waves in a Non-Ideal Gas
}

\author{
R.K. ANAND* AND H.C. YADAV \\ Department of Physics, University of Allahabad, Allahabad-211002, India \\ (Received August 1, 2013; in final form January 5, 2016)
}

\begin{abstract}
This work presents the structure of viscous shock front in a non-ideal gas. The analytical expressions for the particle velocity, temperature, pressure and change-in-entropy within the shock transition region are derived taking into consideration the Landau and Lifshitz equation of state for non-ideal gas. The effects on the structure of shock front due to the variations of the coefficient of viscosity, Mach number, adiabatic exponent and parameter of non-ideality of the gas are investigated. The model developed in the paper is valid only for small values of Mach number $M$ i.e., $M<2.5$.
\end{abstract}

DOI: 10.12693/APhysPolA.129.28

PACS: 52.35.Tc, 47.55.Kf, 47.40.- $\mathrm{x}$

\section{Introduction}

Shock waves arise in a wide range of physical phenomena such as gas dynamics, nuclear explosions, shallow water flows, supernovae, stellar winds, traffic flows, quantum fluids, and many others. The theory of shock waves has a rich history beginning with the fundamental contributions by Riemann in the mid of the 19th century. In fact, all natural fluids admit some compressibility and therefore support shock waves. Shock waves can only develop in a medium which behaves like a fluid. Shock waves may be produced in fluids such as sea water by a variety of natural and artificial mechanisms. The flow parameters such as pressure, density, temperature, particle velocity and entropy change very rapidly in the thin transition layer, through which the gas passes from its initial state of thermodynamic equilibrium into its final, also equilibrium state. Here, the thermodynamic equilibrium inside this region is called the shock front and it can be substantially disturbed. Therefore, in studying the internal structure of a shock front it is necessary to consider the dissipative processes due to viscosity (internal friction) and thermal conduction. The study of the internal structure of shock front has its importance for many reasons. At first this problem attracted attention as purely a theoretical one, the solution of which describes the physical mechanism of shock compression, as a truly remarkable phenomenon in gas dynamics and also in understanding the various processes which take place in gases at high temperatures, as for example, vibrational excitation in molecules, molecular dissociation, chemical reactions, ionization, and radiation. Obviously, the theoretical consideration of the structure of shock front permits one to deduce from the experimental data a good deal of valuable information about the rates of these processes.

\footnotetext{
* corresponding author; e-mail anand.rajkumar@rediffmail.com
}

In the beginning, the dissipative processes due to viscosity and thermal conduction were investigated by Rankine [1], Rayleigh [2] and Taylor [3]. Landau and Lifshitz [4] presented the complete solution for estimating the thickness of shock front in an ideal gas. An exact analytic solution for a shock wave of arbitrary strength was first obtained by Becker [5] and subsequently investigated by Morduchow and Libby [6]. This simple and graphic solution describes all the physical laws governing the structure of shock front in an ideal gas. Tamm [7] and Mott-Smith [8] applied the Boltzmann kinetic equation to the problem of the structure of shock front in a perfect gas. The structure of shock front in a liquid was investigated by Hoover [9]. Sakurai $[10,11]$ refined the Mott-Smith method on the basis of a hard sphere model for the molecular interaction, and predicted that the thickness of shock front approaches a finite limit as the strength of shock wave tends to infinity. Several other authors [12-15] developed the Mott-Smith method and treated the shock front on the basis of Boltzmann's equation. Zel'dovich and Raizer [16] studied the entropy production due to the propagation of plane shock waves in a viscous gaseous medium. The propagation of plane, cylindrical and spherical shock waves in a viscous medium was investigated by Yadav and Anand [17]. Recently, Anand and Yadav [18] have studied the structure of MHD shock waves in a viscous non-ideal gas.

It is assumed, in general, that the gaseous medium behaves as an ideal gas following the equation of state $P V=$ $n R T$, at low density and low temperature. The particles of the medium travel along the straight paths and collide with each other occasionally due to their thermal motions, thus the mean free path time becomes larger than the inter-particle interaction time. But at high density and temperature, mean free path decreases and thus the inter-particle interaction time increases. Under such condition, the mean energy of inter-particle interaction increases and when this energy becomes comparable with the mean kinetic energy of thermal motion, the gaseous medium begins to deviate from the behavior of an ideal gas. Now, the effect arises due to non-ideality of the gas 
becomes important. Thus, the study of shock waves in a non-ideal gas is of great interest both from the mathematical as well as the physical point of view due to its applications in a variety of fields such as microfluids, nuclear science, geophysics, plasma physics, aerodynamics, astrophysics and interstellar medium structure. The contribution of Anisimov and Spiner [19], Steiner and Gretler [20], Kjellander et al. [21], Anand [22-24] and many others is remarkable for the study of shock waves in non-ideal gaseous media. Wu and Roberts [25] and Roberts and $\mathrm{Wu}[26]$ studied the problem of a spherical implosion by considering a simplified form of Van der Waals' equation of state. Srivastava and Singh [27] studied the flow field behind the normal shock of variable strength in a viscous and heat conducting medium with the application of an exact solution representing the progressive waves. The pressure shocks in the relativistic viscous heat conducting fluids were investigated by Ganagi and Gopalakrishana [28], considering the Eckart model and neglecting gravitational effects. Ben-Dor [29] investigated the classical three-shock theory of von Neumann with viscous effects in shock tubes and waves. The effects of thermal conductivity and viscosity on shock waves in argon were studied by Henderson et al. [30]. Simeonides [31] studied viscous effect in the hypersonic flow. Huang et al. [32] investigated viscous shock waves in a compressible gas. Elizarova et al. [33] calculated the structure of shock wave for argon and helium using NS approach and compared the results with experimental data. Thompson et al. [34] estimated the thickness of shock front for a stationary shock in an ideal gas by equating the internal entropy production to the entropy increase found from the Rankine-Hugoniot equation.

In determining the structure and thickness of shock front, the dissipative processes such as viscosity and thermal conduction play key role within the shock front as the gradients are very or almost infinitely steep there. It is also worth mentioning that the viscosity plays a major role in the mechanism of shock compression rather than the heat conduction, as the viscosity of the gas causes the scattering of directed momentum of incident gas and the conversion of kinetic energy of directed molecular motion into the kinetic energy of random motion, i.e., the conversion of mechanical energy into heat energy. It is notable that the heat conduction only indirectly affects the conversion of mechanical energy due to the redistribution of the pressure. The thickness of viscous shock front is proportional to the coefficient of viscosity which in turn is proportional to the molecular mean free path $l$. In the limiting case $l \rightarrow 0$, the hydrodynamics of real fluids becomes, in the continuous flow regions, the hydrodynamics of an ideal fluid. Thus, in this limit the shock front is treated as a mathematical surface. In this case, the gradients of all the flow variables across the front tend to infinity as $1 / l$ but their jumps remain finite [16]. In nature, the viscous transport coefficients cannot vanish but must have lower bounds and thus, the small viscous corrections are always needed in the fluid dynamics.
It is notable that the most of the prior studies have remained focused on the viscous shocks in an ideal gas. However, it is well known that the viscosity has an important role in the characterization of the shocks in nonideal gas rather than in an ideal gas. To the authors' best knowledge, so far there is no paper reporting the structure of viscous shock front in non-ideal gas considering the Landau and Lifshitz equation of state [35] for a non-ideal gas. For this purpose, a model is proposed to provide a simplified, complete treatment for the structure of plane viscous shock waves in a non-ideal gas using the Landau and Lifshitz equation of state [35]. It is to be noted that the equation of state is valid for the case of propagation of the shock waves for upstream molecular gases and the equation of state may not be valid for downstream gases if destruction of molecules in downstream due to shock heating is taken into account. The analytical expressions for the flow variables such as the particle velocity $\eta$, the temperature $T / T_{0}$, the pressure $p / p_{0}$ and the change-in-entropy $\Delta S / \Gamma$ within the shock transition region are obtained in terms of the Mach number $M$, coefficient of viscosity $\mu$, adiabatic exponent $\gamma$ and parameter of non-ideality $b \rho_{0}$ of the gas. It is worth mentioning that the validity of the model presented in this paper is only for small values of the Mach number $M$, i.e., $M<2.5$ as the effect of thermal conductivity becomes negligible for above mentioned small values of $M$ [36]. It is remarkable that the present model appropriately makes obvious the effects due to an increase in (i) the distance from the origin, $\mathrm{O}$, (ii) the parameter of non-ideality of the gas, (iii) the coefficient of viscosity, (iv) the Mach number, and (v) the adiabatic exponent, on the particle velocity or inverse of density, the temperature, the pressure and the change-in-entropy within the viscous shock front in non-ideal gas. The results are discussed and compared with those for the case of an ideal gas. Thus, the results provided a clear picture of whether and how the parameter of non-ideality of the gas, coefficient of viscosity, the Mach number, and the adiabatic exponent affect the thickness of shock front as well as the variations in the flow variables within the plane shock transition region.

The rest of the paper is organized as follows: Sect. 2 describes the general assumptions and notations, equation of state for non-ideal gas and boundary condition. In Sect. 3 the general non-dimensional forms of the analytical expressions for the distribution of the flow variables are presented. A brief discussion of the results is presented in Sect. 4. The findings are concluded in Sect. 5 with details on which effects were accounted for and which were not.

\section{Basic equations and boundary condition}

The unsteady, one-dimensional flow field in a viscous non-ideal gas is a function of two independent variables: the time $t$ and the space coordinate $r$. In order to get some essential features of the structure of shock front, it is assumed that the gaseous flow has a finite viscosity and 
zero thermal conductivity, and the equilibrium-flow condition is maintained in the flow field. The conservation equations governing the flow of a one-dimensional, viscous, non-ideal gas under an equilibrium condition can be expressed conveniently in Eulerian coordinates as follows:

$$
\begin{aligned}
& \frac{\partial \rho}{\partial t}+\rho \frac{\partial u}{\partial r}+u \frac{\partial \rho}{\partial r}=0, \\
& \frac{\partial(\rho u)}{\partial t}+\frac{\partial\left(p+\rho u^{2}-q\right)}{\partial r}=0, \\
& \frac{\partial\left(\rho e+\rho u^{2} / 2\right)}{\partial t}+\frac{\partial\left[\rho u\left(e+u^{2} / 2\right)+p u-q u\right]}{\partial r}=0,
\end{aligned}
$$

where $\rho(r, t), u(r, t), p(r, t), q(r, t)$ and $e(r, t)$ are density, particle velocity, pressure, viscous stress tensor, and internal energy per unit mass, respectively. $r$ is the position coordinate with respect to the origin, $O$, measured in the direction normal to the shock front and $t$ is the time coordinate.

Landau and Lifshitz [35] gave the equation of state for a non-ideal gas as

$$
p=\Gamma \rho T\left[1+\rho C_{1}(T)+\rho^{2} C_{2}(T)+\ldots\right]
$$

where $\Gamma$ is the gas constant, $p, \rho$ and $T$ are the pressure, density, and temperature of the non-ideal gas, respectively, and $C_{1}(T)$ and $C_{2}(T)$ are virial coefficients. The first term in the expansion corresponds to an ideal gas. The second term is obtained by taking into account the interaction between the pairs of molecules, and subsequent terms must involve the interaction between the groups of three, four, etc., molecules. In the high temperature range, the coefficients $C_{1}(T)$ and $C_{2}(T)$ tend to constant values equal to $b$ and $(5 / 8) b^{2}$, respectively. For gases $b \rho \ll 1, b$ being the internal volume of the molecules, and therefore, it is sufficient to consider the above equation of state [19] in the form

$$
p=\Gamma \rho T(1+b \rho) \text {. }
$$

The internal energy $e$ per unit mass of the non-ideal gas is given as (see Ref. [22])

$$
e=p / \rho(\gamma-1)(1+b \rho),
$$

where $\gamma$ is the adiabatic index. Equation (5) implies that

$$
C_{p}-C_{v}=\Gamma\left(1+b^{2} \rho^{2} /(1+2 b \rho)\right) \cong \Gamma,
$$

neglecting the second and higher powers of $b \rho$. Here $C_{p}$ and $C_{v}$ are the specific heats of the gas at constant pressure and constant volume, respectively. Using the first law of thermodynamics and Eqs. (4) and (5), we obtain the isentropic exponent $\Gamma^{*}=\gamma(1+2 b \rho) /(1+b \rho)$, neglecting the second and higher powers of $b \rho$. The isentropic velocity of sound $a$, in a non-ideal gas is given by

$$
a^{2}=\Gamma^{*} p / \rho \text {. }
$$

The viscous stress tensor $(q)$ is given by

$$
q=(4 / 3) \mu(\mathrm{d} u / \mathrm{d} r),
$$

where $\mu$ is the coefficient of viscosity, which is assumed to be independent of the temperature for simplicity.

In the coordinate system with a stationary shock front, the shock strength is practically unchanged during the small time interval $\Delta t$, required to travel a distance of the order of the shock front thickness, and for this reason, in the flow Eqs. (1)-(3), the term containing the partial derivative with respect to time $(\partial / \partial t)$ can be dropped and the partial derivative $(\partial / \partial r)$ can be replaced by the total derivative $(\mathrm{d} / \mathrm{d} r)$. Thus, Eqs. (1)-(3) can be written as

$$
\begin{aligned}
& u \frac{\mathrm{d} \rho}{\mathrm{d} r}+\rho \frac{\mathrm{d} u}{\mathrm{~d} r}=0, \\
& \frac{\mathrm{d}\left(p+\rho u^{2}-q\right)}{\mathrm{d} r}=0, \\
& \frac{\mathrm{d}\left[\rho u\left(e+u^{2} / 2\right)+p u-q u\right]}{\mathrm{d} r}=0 .
\end{aligned}
$$

The boundary condition on the solutions of the above differential Eqs. (8)-(10) requires that the gradients of the flow variables must vanish ahead of the shock front (at $r=+\infty$ ) as well as behind the shock front (at $r=$ $-\infty)$. With these limits, the initial flow variables designated by the subscript " 0 " are $p_{0}, \rho_{0}, u_{0}$, and the final flow variables with no subscript are $p, \rho, u$. If the shock front is moving with velocity $U$, then in the coordinate system fixed with the shock front, the initial particle velocity $u_{0}$ will be

$$
u_{0}=U \text {. }
$$

\section{Exact solutions for the flow variables}

In order to obtain the exact solutions for the flow variables, we integrate Eqs. (8)-(10) using the boundary condition given by Eq. (11) in the equilibrium state and then using Eq. (5) we get

$$
\begin{aligned}
& \rho=\rho_{0} U / u, \\
& p=p_{0}+q+\rho_{0} U^{2}-\rho u^{2}, \\
& p u /(\gamma-1)(1+b \rho)+\rho u^{3} / 2+p u-q u= \\
& \quad p_{0} U /(\gamma-1)\left(1+b \rho_{0}\right)+\rho_{0} U^{3} / 2+p_{0} U .
\end{aligned}
$$

Using Eqs. (12) and (13), Eq. (14) can be written as

$$
\gamma p_{0} \eta / \rho_{0} U^{2}+\gamma \eta(1-\eta)-b \rho_{0} q / \rho_{0} U^{2}-b \rho_{0}(1-\eta)
$$

$+(\gamma-1) \eta^{2} / 2+q U \eta / \rho_{0} U^{3}=\gamma p_{0} / \rho_{0} U^{2}+(\gamma-1) / 2 .(15)$ Let us define two non-dimensional quantities called particle velocity $\eta$ and shock strength $M$ as

$$
\eta=u / U=\rho_{0} / \rho \text { and } M=U / a_{0},
$$

where $a_{0}^{2}=\gamma p_{0} \delta / \rho_{0}$ is the speed of sound in the unperturbed state, and $\delta=\left(1+2 b \rho_{0}\right) /\left(1+b \rho_{0}\right)$. Substituting Eqs. (7) and (16) into Eq. (15), we get

$$
a_{1} \eta^{2}+b_{1} \eta+c_{1}=g_{1}\left(\eta-b \rho_{0}\right) \mathrm{d} \eta / \mathrm{d} r
$$

where

$$
\begin{aligned}
& a_{1}=(\gamma+1) / 2, \quad b_{1}=-\left(\gamma+b \rho_{0}+1 / \delta M^{2}\right), \\
& c_{1}=\left(b \rho_{0}+1 / \delta M^{2}+(\gamma-1) / 2\right), \\
& g_{1}=4 \mu / 3 M\left(\gamma p_{0} \rho_{0} \delta\right)^{1 / 2} .
\end{aligned}
$$

Eq. (17) is quadratic in $\eta$. In equilibrium state, there are no gradients in the flow variables. Thus, the condition for the equilibrium state is

$$
\mathrm{d} \eta / \mathrm{d} r=0 \text { with } \eta=\eta_{\text {eq }} .
$$


For $\eta_{\mathrm{eq}}$, the two solutions of Eq. (17) are

$$
\eta_{\mathrm{eq}}=\left[-b_{1} \pm \sqrt{\left(b_{1}^{2}-4 a_{1} c_{1}\right)}\right] / 2 a_{1} .
$$

We denote first equilibrium state by $\eta_{1}$ with positive sign and second equilibrium state by $\eta_{2}$ with negative sign as

$$
\eta_{1,2}=\left[-b_{1} \pm \sqrt{\left(b_{1}^{2}-4 a_{1} c_{1}\right)}\right] / 2 a_{1} .
$$

Now integrating Eq. (17) and using Eq. (18), we get

$$
r=\left(g_{1} / a_{1}\right)\left[A \log \left(\eta-\eta_{1}\right)+B \log \left(\eta-\eta_{2}\right)\right]+C^{\prime},
$$

where $C^{\prime}$ is the constant of integration.

For searching the value of the constant of integration $C^{\prime}$, we choose the origin, $O$, at the point of inflection of velocity profile which is defined by the condition $\mathrm{d}^{2} \eta / \mathrm{d} r^{2}=0$. Using this condition in Eq. (17), we get

$$
\mathrm{d}^{2} \eta / \mathrm{d} r^{2}=a_{1} \eta^{2}-2 a_{1}\left(b \rho_{0}\right) \eta-\left(c_{1}+b_{1} b \rho_{0}\right)=0 .
$$

The solution of Eq. (20) which gives us the point of inflection, is

$$
\eta_{\text {in }}^{\prime}=b \rho_{0} \pm\left\{\left(b \rho_{0}\right)^{2}+\left(c_{1}+b_{1} b \rho_{0}\right) / a_{1}\right\}^{1 / 2} .
$$

Using this condition, the constant of integration $C^{\prime}$ in Eq. (19) becomes

$$
C^{\prime}=-\left(g_{1} / a_{1}\right)\left[A \log \left(\eta_{\text {in }}^{\prime}-\eta_{1}\right)+B \log \left(\eta_{\text {in }}^{\prime}-\eta_{2}\right)\right] .(22
$$

Substituting Eq. (22) into Eq. (19), finally we get the exact solution for the particle velocity with respect to the distance as

$$
\begin{aligned}
r & =\left(g_{1} / a_{1}\right)\left[A \log \left(\left(\eta-\eta_{1}\right) /\left(\eta_{\text {in }}^{\prime}-\eta_{1}\right)\right)\right. \\
& \left.+B \log \left(\left(\eta-\eta_{2}\right) /\left(\eta_{\text {in }}^{\prime}-\eta_{2}\right)\right)\right],
\end{aligned}
$$

where

$$
A=\left(\eta_{1}-b \rho_{0}\right) /\left(\eta_{1}-\eta_{2}\right)
$$

and

$$
B=\left(\eta_{2}-b \rho_{0}\right) /\left(\eta_{2}-\eta_{1}\right) .
$$

Equation (23) shows that the particle velocity depends on the distance $r$ within the shock transition region.

Using Eqs. (5), (7) and (14), the exact solution for the temperature within the shock transition region is obtained as

$$
\begin{aligned}
& T / T_{0}=1+\gamma \delta M^{2}(\gamma-1)\left(1+b \rho_{0}\right) \\
& \quad \times\left[\eta^{2} / 2-\left(1+1 / \gamma \delta M^{2}\right) \eta+\left(1 / 2+1 / \gamma \delta M^{2}\right)\right] .
\end{aligned}
$$

The above Eq. (24) shows that the temperature depends on the particle velocity. From Eqs. (23) and (24), we can calculate the temperature within the shock transition region with respect to the distance $r$. Using Eqs. (7), (12) and (17) into Eq. (13), the exact solution for the pressure within the shock transition region can be written as

$$
\begin{aligned}
& p / p_{0}=1+\gamma \delta M^{2}[(1-\eta) \\
& \left.\quad+\left(a_{1} \eta^{2}+b_{1} \eta+c_{1}\right) /\left(\eta-b \rho_{0}\right)\right] .
\end{aligned}
$$

Thus, Eqs. (23) and (25) simultaneously describe the dependence of the pressure across the shock front on the distance. Further, we can write an explicit formula for the change-in-entropy $\Delta S / \Gamma$ across the shock of an arbitrary strength in non-ideal gas as

$$
\Delta S / \Gamma=(\gamma-1)^{-1} \log \left(T / T_{0}\right)+\log \eta-\eta^{-1} b \rho_{0} .
$$

Thus, the entropy production across the shock front is easily obtained by substituting Eqs. (23) and (24) into Eq. (26).

\section{Results and discussion}

In the present paper exact solution for the structure of viscous shock front in non-ideal gas was obtained and further the effects due to the parameter of non-ideality of the gas on the flow variables within the shock transition region were explored. The equation of state for non-ideal gas was considered as given by Landau and Lifshitz [35]. The non-dimensional analytical expressions for the particle velocity $\eta$, the temperature $T / T_{0}$, the pressure $p / p_{0}$ and the change-in-entropy $\Delta S / \Gamma$ within the shock transition region are given by Eqs. (23), (24), (25) and (26), respectively. These analytical expressions were derived by assuming that the disturbances due to the reflections, wave interactions in the wake, etc., do not overtake the shock waves. The expressions for the particle velocity, the temperature, the pressure and the change-in-entropy are functions of the distance $r$, the Mach number $M$, the coefficient of viscosity $\mu$, the adiabatic exponent $\gamma$ and the parameter of non-ideality $b \rho_{0}$ of the gas. Therefore, the values of the constant parameters are taken to be $M=1.1,1.5,2.0, \mu=15 \times 10^{-6}, 17.2 \times 10^{-6}$, $20 \times 10^{-6} \mathrm{~Pa} \mathrm{~s}, b \rho_{0}=0,0.0625,0.1250,0.1875,0.2500$, $\gamma=1.33,1.4,1.66$, initial pressure $p_{0}=0.9$ bar and initial density $\rho_{0}=1.20 \mathrm{~kg} / \mathrm{m}^{3}$ for the purpose of numerical computations. The value $b \rho_{0}=0$, corresponds to the case of a perfect gas. The numerical estimations of the flow variables within the shock transition region are carried out using MATLAB code.

It is notable that the thickness of shock front increases with increase in the coefficient of viscosity, however, it decreases with increase in the shock strength. It is remarkable that the thickness of shock front slightly decreases with increase in the parameter of non-ideality of the gas for $M=1.5$ and 2.0, whereas it increases for $M=1.1$ (see Table I).

The variations of the particle velocity, the temperature, the pressure and the change-in-entropy distribution within shock transition region with distance $r$ for $M=2, \gamma=1.33, p_{0}=0.9$ bar, $\rho_{0}=1.20 \mathrm{~kg} / \mathrm{m}^{3}$, $\mu=15 \times 10^{-6} \mathrm{~Pa} \mathrm{~s}$ and various values of $b \rho_{0}$ are shown in Fig. 1. It is found that within the shock transition region around the point of inflection the rate of change of the flow variables increases for $b \rho_{0}=0$ (ideal gas), however, it decreases as the parameter of non-ideality of the gas increases. This behavior of the flow variables with distance $r$, especially for the case of $b \rho_{0}=0.2500$ differs greatly from the case of an ideal gas for which $b \rho_{0}=0$. The spreading of flow variables is slightly large for an ideal gas than that for non-ideal gas and also it decreases as the parameter of non-ideality of the gas increases. Thus, the thickness of shock front is maximal for $b \rho_{0}=0$ and minimum for $b \rho_{0}=0.2500$. It is also notable that the entropy production within the shock front 
TABLE I

The thickness of viscous shock front in non-ideal gas for $\gamma=1.4, p_{0}=0.9$ bar and $\rho_{0}=1.20 \mathrm{~kg} / \mathrm{m}^{3}$.

\begin{tabular}{|c|c|c|c|c|c|}
\hline $\begin{array}{c}\mu \\
{[\mathrm{Pa}}\end{array}$ & $M$ & $b \rho_{0}$ & $\begin{array}{l}r_{1} \\
{[\mathrm{~m}]}\end{array}$ & $\begin{array}{c}r_{2} \\
{[\mathrm{~m}]}\end{array}$ & $\begin{array}{c}r=r_{2}-r_{1} \\
{[\mathrm{~m}]}\end{array}$ \\
\hline \multirow{9}{*}{$15 \times 10^{-6}$} & \multirow{3}{*}{1.1} & 0 & -0.0008317 & 0.0007005 & 0.0015322 \\
\hline & & 0.1250 & -0.00102 & 0.0007635 & 0.0017835 \\
\hline & & 0.2500 & -0.001426 & 0.001316 & 0.002742 \\
\hline & \multirow{3}{*}{1.5} & 0 & -0.0002317 & 0.0001143 & 0.000346 \\
\hline & & 0.1250 & -0.0002198 & 0.0001115 & 0.0003313 \\
\hline & & 0.2500 & -0.0002054 & 0.0001187 & 0.0003241 \\
\hline & \multirow{3}{*}{2.0} & 0 & -0.0001185 & 0.00004615 & 0.00016465 \\
\hline & & 0.1250 & -0.0001162 & 0.00004431 & 0.00016051 \\
\hline & & 0.2500 & -0.0001102 & 0.000045 & 0.0001552 \\
\hline \multirow{9}{*}{$17.2 \times 10^{-6}$} & \multirow{3}{*}{1.1} & 0 & -0.0009537 & 0.0008032 & 0.0017569 \\
\hline & & 0.1250 & -0.00117 & 0.0008755 & 0.0020455 \\
\hline & & 0.2500 & -0.001635 & 0.001509 & 0.003144 \\
\hline & \multirow{3}{*}{1.5} & 0 & -0.0002657 & 0.0001311 & 0.0003968 \\
\hline & & 0.1250 & -0.000252 & 0.0001278 & 0.0003798 \\
\hline & & 0.2500 & -0.0002355 & 0.0001361 & 0.0003716 \\
\hline & \multirow{3}{*}{2.0} & 0 & -0.0001359 & 0.00005291 & 0.00018881 \\
\hline & & 0.1250 & -0.0001333 & 0.0000508 & 0.0001841 \\
\hline & & 0.2500 & -0.0001264 & 0.00005161 & 0.00017801 \\
\hline \multirow{9}{*}{$20 \times 10^{-6}$} & \multirow{3}{*}{1.1} & 0 & -0.001109 & 0.000934 & 0.002043 \\
\hline & & 0.1250 & -0.001361 & 0.001018 & 0.002379 \\
\hline & & 0.2500 & -0.001901 & 0.001755 & 0.003656 \\
\hline & \multirow{3}{*}{1.5} & 0 & -0.0003089 & 0.0001524 & 0.0004613 \\
\hline & & 0.1250 & -0.0002931 & 0.0001486 & 0.0004417 \\
\hline & & 0.2500 & -0.0002738 & 0.0001583 & 0.0004321 \\
\hline & \multirow{3}{*}{2.0} & 0 & -0.000158 & 0.00006153 & 0.00021953 \\
\hline & & 0.1250 & -0.000155 & 0.00005908 & 0.00021408 \\
\hline & & 0.2500 & -0.000147 & 0.00006001 & 0.00020701 \\
\hline
\end{tabular}

is slightly large for $b \rho_{0}=0$ than for $b \rho_{0}=0.2500$. It is worth mentioning that the plots for the particle velocity, the temperature and the pressure are distinguishable towards the upstream side i.e., ahead of the point of inflection, however, these plots are not so much distinguishable towards the downstream side. On the other hand, the plots for change-in-entropy are more distinguishable towards both the downstream and upstream sides.

The variations in the flow variables with respect to the distance for $M=2, \gamma=1.33, p_{0}=0.9$ bar, $\rho_{0}=1.20 \mathrm{~kg} / \mathrm{m}^{3}$ and various values of $\mu$ and $b \rho_{0}$ are shown in Fig. 2. It is observed that the spreading of the flow variables increases with increasing value of the coefficient of viscosity for both cases of ideal and non-ideal gases. It is notable that the spreading of the flow variables is slightly large in case of an ideal gas. Thus, the thickness of shock front is slightly small for higher values of $b \rho_{0}$. It is found that within the shock transition region, the rate of change of the flow variables around the point of inflection decreases with increasing value of the coefficient of viscosity of the gas. It is also obvious from Fig. 2 that the entropy production within a shock front is slightly large for $b \rho_{0}=0$ than for $b \rho_{0}=0.2500$.
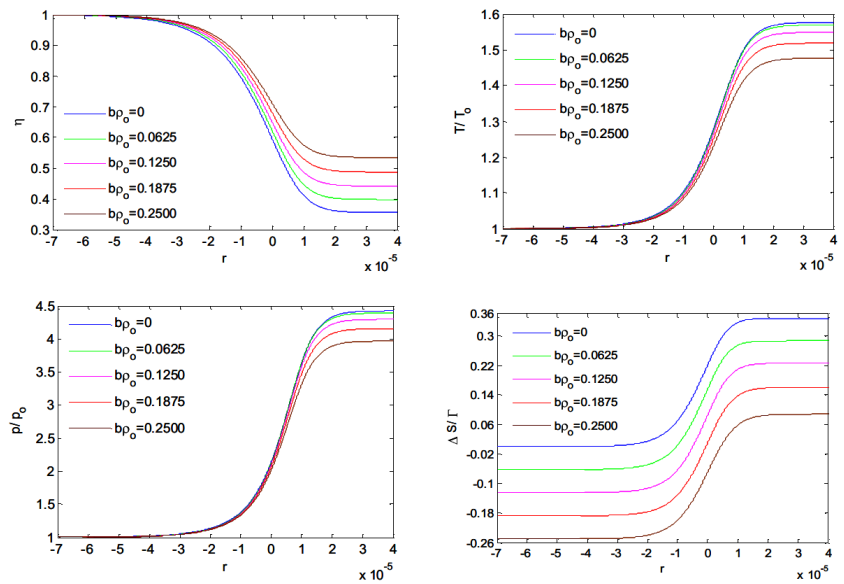

Fig. 1. The variations of particle velocity, temperature, pressure and change-in-entropy distribution within shock transition region with distance $r$ for $M=2, \gamma=$ 1.33, $p_{0}=0.9$ bar, $\rho_{0}=1.20 \mathrm{~kg} / \mathrm{m}^{3}, \mu=15 \times 10^{-6} \mathrm{~Pa} \mathrm{~s}$ and various values of $b \rho_{0}$.
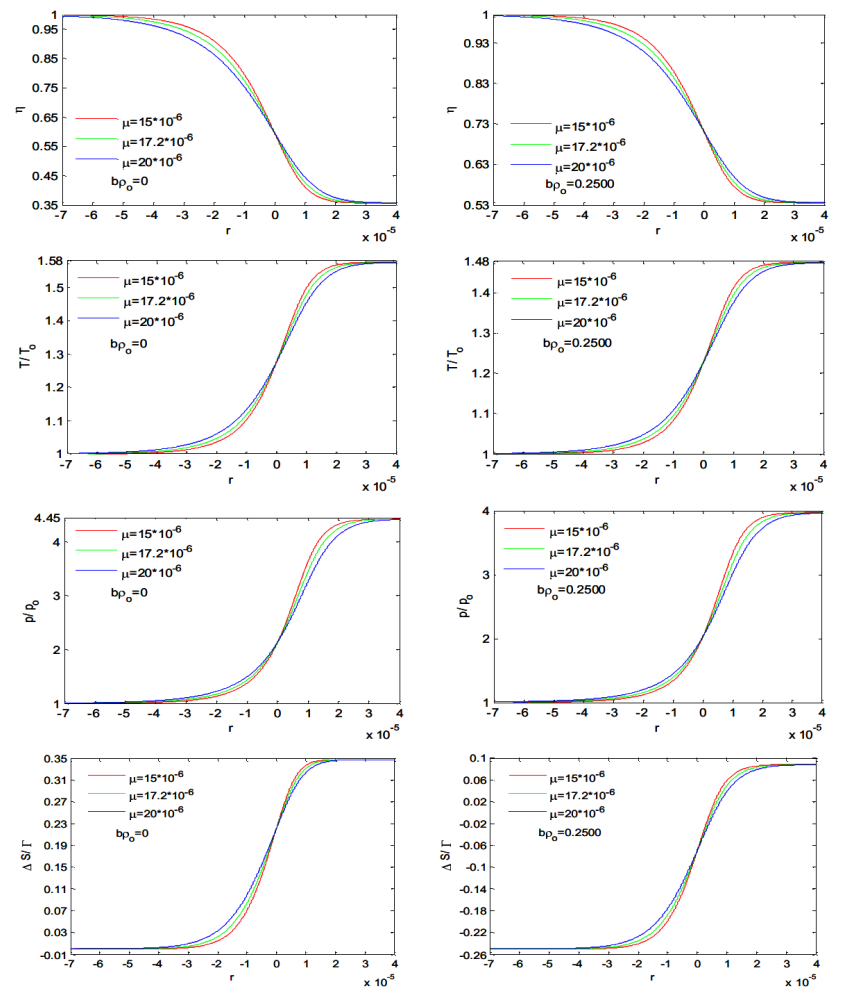

Fig. 2. The variations of particle velocity, temperature, pressure and change-in-entropy distribution within shock transition region with distance $r$ for $M=2$, $\gamma=1.33, p_{0}=0.9$ bar, $\rho_{0}=1.20 \mathrm{~kg} / \mathrm{m}^{3}$ and various values of $\mu$ and $b \rho_{0}$.

Thus, the thickness of shock front in an ideal gas is slightly large.

The variations of the flow variables within shock transition region with respect to the distance for $\gamma=1.33$, $p_{0}=0.9$ bar, $\rho_{0}=1.20 \mathrm{~kg} / \mathrm{m}^{3}, \mu=15 \times 10^{-6} \mathrm{~Pa} \mathrm{~s}$ and various values of $M$ and $b \rho_{0}$ are shown in Fig. 3 . 

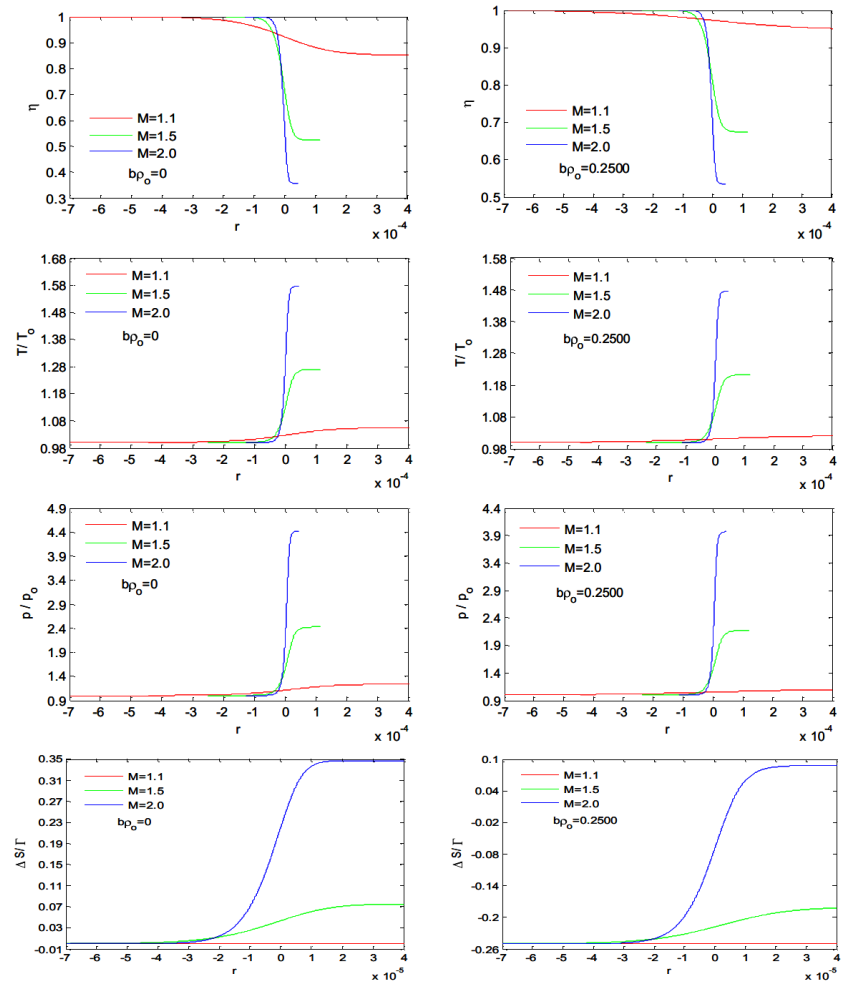

Fig. 3. The variations of particle velocity, temperature, pressure and change-in-entropy distribution within shock transition region with distance $r$ for $\gamma=1.33$, $p_{0}=0.9$ bar, $\rho_{0}=1.20 \mathrm{~kg} / \mathrm{m}^{3}, \mu=15 \times 10^{-6} \mathrm{~Pa} \mathrm{~s}$ and various values of $M$ and $b \rho_{0}$.

It is notable that the spreading of flow variables is small for $M=2$ and it increases with decreasing value of $M$. Thus, the thickness of shock front decreases with increasing value of $M$ for both cases of ideal and non-ideal gases. It is notable that the variations of the particle velocity, temperature and pressure within the shock front region are slightly small in non-ideal gas. Thus, the thickness of shock front in non-ideal gas is slightly small for every value of $M$. The entropy production within the shock front region is slightly large for $b \rho_{0}=0$ than for $b \rho_{0}=0.2500$. It is notable that the plots for all flow variables are distinguishable towards the upstream side whereas these plots are not so much distinguishable towards the downstream side.

The variations of the flow variables with respect to the distance for $M=2, p_{0}=0.9$ bar, $\rho_{0}=1.20 \mathrm{~kg} / \mathrm{m}^{3}$, $\mu=15 \times 10^{-6} \mathrm{~Pa} \mathrm{~s}$ and various values of $\gamma$ and $b \rho_{0}$ are shown in Fig. 4. It is observed that the spreading of flow variables is small for $\gamma=1.33$ and it increases with increasing value of $\gamma$. Thus, the thickness of shock front increases with increasing value of $\gamma$ for both cases of ideal and non-ideal gases. The variations of the particle velocity, temperature and pressure within the shock transition region are slightly small in the non-ideal gas. Thus, the thickness of shock front is slightly small in non-ideal gas for every value of $\gamma$. It is noteworthy that the entropy production within the shock front region is slightly large
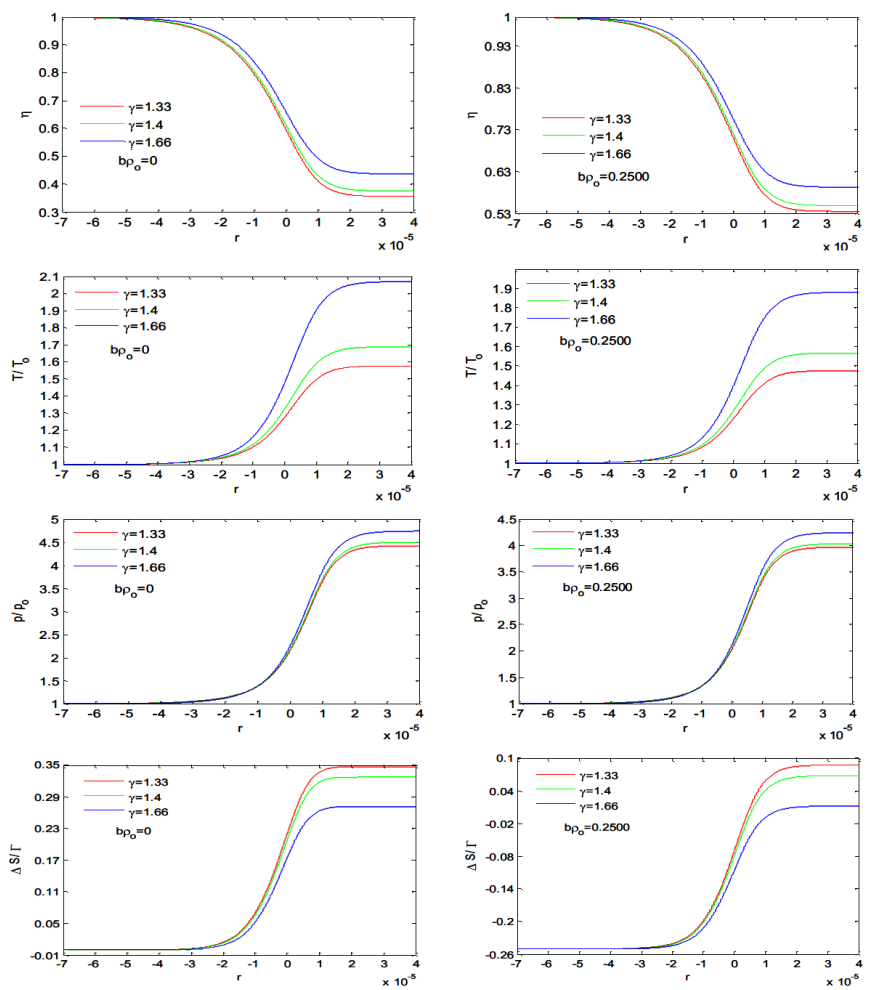

Fig. 4. The variations of particle velocity, temperature, pressure and change-in-entropy distribution within shock transition region with distance $r$ for $M=2$, $p_{0}=0.9$ bar, $\rho_{0}=1.20 \mathrm{~kg} / \mathrm{m}^{3}, \mu=15 \times 10^{-6} \mathrm{~Pa} \mathrm{~s}$ and various values of $\gamma$ and $b \rho_{0}$.

for $b \rho_{0}=0$ than for $b \rho_{0}=0.2500$. It is also notable that the plots for all flow variables are distinguishable towards the upstream side but are not so much distinguishable towards the downstream side.

\section{Conclusions}

The investigations made in the present paper are intended to contribute to the understanding of the structure of viscous shock front in real gases [19, 22, 35], by giving, for the first time, the full exact solutions for the flow field within the shock transition region. The analysis presented in the paper shows the fundamental role played by viscosity and non-ideality of the gas in determining the structure of shock front. The following conclusions may be drawn from the findings of the current analysis:

1. The thickness of shock front decreases with increasing value of the parameter of non-ideality of the gas and the Mach number.

2. The thickness of shock front increases with increasing value of the coefficient of viscosity and adiabatic index of the gas.

3. The entropy production within viscous shock front region decreases with increasing value of the parameter of non-ideality of the gas. 
It is worth mentioning that the effect of thermal conductivity can be neglected for small values of Mach number $M$ thus, the present model remains valid only for $M<2.5$.

\section{Acknowledgments}

The anonymous referees are greatly appreciated for their comments and suggestions.

\section{References}

[1] W.J.M. Rankine, Trans. R. Soc. Lond. 160, 277 (1870).

[2] J. Rayleigh, Proc. R. Soc. Lond. A 84, 247 (1910).

[3] G.I. Taylor, Proc. R. Soc. Lond. A 84, 371 (1910).

[4] L.D. Landau, E.M. Lifshitz, Fluid Mechanics, Course of Theoretical Physics, Vol. 6, Pergamon Press, Oxford 1987.

[5] R. Becker, Z. Phys. 8, 321 (1922).

[6] M. Morduchow, P.A. Libby, J. Aeronaut. Sci. 16, 674 (1949).

[7] I.E. Tamm, Tr. Fiz. Inst. Akad. Nauk SSSR 29, 239 (1965).

[8] H.M. Mott-Smith, Phys. Rev. 82, 885 (1951).

[9] W.G. Hoover, Phys. Rev. Lett. 42, 1531 (1979).

[10] A. Sakurai, Fluid Mech. 3, 255 (1957).

[11] A. Sakurai, A note on Mott-Smith's solution of the Boltzmann equation for a shock wave, II. Research Report, Vol. 6, Tokyo Electrical Engineering College, Tokyo 1958, p. 49.

[12] W.A. Gustafson, Phys. Fluids 3, 732 (1960).

[13] C. Muckenfuss, Phys. Fluids 3, 320 (1960).

[14] S. Ziering, F. Ek, Phys. Fluids 4, 765 (1961).

[15] P. Glansdorff, Phys. Fluids 5, 371 (1962).

[16] Ya.B. Zel'dovich, Yu.P. Raizer, Physics of Shock Waves and High Temperature Hydrodynamics Phenomena, Dover Publ., New York 2002.

[17] H.C. Yadav, R.K. Anand, Phys. Scr. 83, 065402 (2011).
[18] R.K. Anand, H.C. Yadav, Theor. Comput. Fluid Dyn. 28, 369 (2014).

[19] S.I. Anisimov, O.M. Spiner, J. Appl. Math. Mech. 36, 883 (1972).

[20] H. Steiner, W. Gretler, Phys. Fluids 6, 2154 (1994).

[21] M. Kjellander, N. Tillmark, N. Apazidis, Phys. Fluids 22, 116102 (2010).

[22] R.K. Anand, Astrophys. Space Sci. 342, 377 (2012).

[23] R.K. Anand, Wave Motion 50, 1003 (2013).

[24] R.K. Anand, Phys. Scr. 87, 065404 (2013).

[25] C.C. Wu, P.H. Roberts, Phys. Lett. A 213, 59 (1996).

[26] P.H. Roberts, C.C. Wu, in: Shock Focusing Effect in Medical Science and Sonoluminescence, Eds. R.C. Srivastava, D. Leutloff, K. Takayama, H. Gronig, Springer, Berlin 2003, p. 1.

[27] S.K. Srivastava, R.K. Singh, Astrophys. Space Sci. 102, 247 (1984).

[28] M.S. Ganagi, A.V. Gopalakrishna, Astrophys. Space Sci. 129, 281 (1987).

[29] G. Ben-Dor, in: Shock Tubes and Waves, Ed. H. Gronig, VCH Verlagsgesellschaft mbH, Technische Hochschule, Aachen, West Germany 1988, p. 527.

[30] F. Henderson, W.Y. Crutchfield, R.J. Virgona, Fluid Mech. 331, 1 (1997).

[31] G. Simeonides, Shock Waves 8, 161 (1998).

[32] F. Huang, A. Matsumura, X. Shi, Commun. Math. Phys. 239, 261 (2003).

[33] T.G. Elizarova, I.A. Shirokov, S. Montero, Phys. Fluids 17, 068101 (2005).

[34] P.A. Thompson, T.W. Stroch, D.S. Lim, Phys. Fluids 26, 48 (1983).

[35] L.D. Landau, E.M. Lifshitz, Statistical Physics, Course of Theoretical Physics, Vol. 5, Pergamon Press, Oxford 1958.

[36] Yu.A. Berezin, G.I. Dudnikova, J. Appl. Mech. Tech. Phys. 13, 139 (1972). 